\title{
Examining the Diagnostic Validity of Autism Measures Among Adults in an Outpatient Clinic Sample
}

\author{
Caitlin M. Conner, PhD, Ryan D. Cramer, MSW, and John J. McGonigle, PhD
}

Background: Previous research has questioned the validity of diagnostic measures for autism spectrum disorder (ASD) among adults. This study examined the correspondence between several measures and clinician diagnosis. Methods: We conducted a retrospective chart review for 93 adults (18-61 years; 72\% male) who received an ASD evaluation at a specialty outpatient clinic. Thirty-one individuals (33\%) in the sample were diagnosed with ASD. We compared participant scores on the Autism Spectrum Quotient (AQ), the Ritvo Autism Asperger's Diagnostic Scale-Revised (RAADS-R), and the Autism Diagnostic Observation Schedule (ADOS) to clinician diagnosis of ASD. We calculated sensitivity, specificity, and area under the curve (AUC) for each measure.

Results: Participants diagnosed with ASD scored significantly higher, on average, on the ADOS than those who were not diagnosed with ASD, but not on the RAADS-R or AQ. The AUC was relatively low for each measure: $\mathrm{ADOS}=0.69$ (95\% confidence interval $[\mathrm{CI}] 0.58-0.81)$, RAADS-R $=0.58$ (95\% CI 0.46-0.72), and AQ=0.40 (95\% CI $0.28-0.52$ ). Sensitivity and specificity of all three measures were in the poor to fair range. When dichotomized at the optimal cutoffs for this sample, the ADOS had a sensitivity of 0.65 and a specificity of 0.76 ; the RAADS-R had a sensitivity of 0.52 and a specificity of 0.73 ; and the AQ had a sensitivity of 0.45 and a specificity of 0.52 .

Conclusions: Results of the study suggest that clinicians should not rely solely on self-report measures or the ADOS when diagnosing adults on the spectrum. Further development of measures is needed, including selfreport measures with higher diagnostic validity, that are sensitive across age, gender, and cognitive functioning, and that differentiate autism from psychiatric diagnoses.

Keywords: diagnosis, adults, ASD, ADOS, self-report

\section{Lay Summary}

Why was this study done?

Diagnosing adults with autism spectrum disorder (ASD) is difficult. Other research has suggested that the few measures that exist for autistic adults may not be very effective for accurate diagnoses. We wanted to see how closely the results of commonly used ASD assessment tools compared with clinical diagnoses in a real-life outpatient setting.

\section{What did the researchers do?}

This study looked at adults who went to an adult ASD outpatient clinic for an initial ASD diagnosis over 3 years. Of these 93 adults, one-third were diagnosed as autistic. As part of the evaluation, all participants completed two commonly used autism screening surveys-the Autism Spectrum Quotient (AQ) and the Ritvo Autism Asperger's Diagnostic Scale-Revised (RAADS-R) - and took part in a semistructured diagnostic interview called the Autism Diagnostic Observation Schedule (ADOS). The clinicians also collected additional information and, when possible, spoke to family members before jointly making a diagnosis. The researchers compared how those diagnosed with ASD and those who were not diagnosed with ASD scored on the AQ, the RAADS-R, and the ADOS.

\section{What were the results of this study?}

Although adults on the spectrum scored higher on average on the ADOS clinical interview than those who did not receive an autism diagnosis, they did not score higher on the AQ and RAADS-R self-report measures. All three of the measures were only moderately effective at showing who would be diagnosed with ASD and who was not.

Western Regional Autism Services, Education, Resources, Training (ASERT) Collaborative, Western Psychiatric Institute and Clinic, University of Pittsburgh Medical Center, Pittsburgh, Pennsylvania. 


\section{What do these findings add to what was already known?}

These results confirm and expand on findings from other prior studies. The findings suggest that ASD diagnostic measures should not be used alone or considered the only source of information when making an initial autism diagnosis in adulthood. What are potential weaknesses in the study?

When studying the accuracy of diagnostic tests, it is best to compare the results of the tests being studied with an "independent gold standard," that is, a test that we know is very good and that is totally separate from the tests being studied. In this case, there is no clear "gold standard," so we had to compare the tests with the next best thing-the clinicians' final decision about whether or not a client has a diagnosis of ASD. It is possible that the clinicians did not make the right diagnosis. Also, the diagnosis was not "independent" of the tests being studied, since the clinicians used the results of the tests to help make the diagnosis. Clinicians only diagnosed about one-third of adults in this study with ASD, whereas previous studies in community clinics have had a higher percentage of adults diagnosed; this factor may have influenced the measures' accuracy. Lastly, clinicians in this study did not assess clients for any other mental health conditions, which may have provided more information about the clients who were not diagnosed with ASD.

How will these findings help autistic adults now or in the future?

The findings from this study suggest that none of these measures are very accurate on their own. Thus, we recommend multiple measures (interviews and questionnaires) should be used together when clinicians diagnose ASD in adulthood. Results of this study also suggest that measures for adults with ASD should be tested in real-world community clinics, so that clinicians and researchers see how the measures perform when used for initial diagnosis in adulthood.

\section{Introduction}

$\mathbf{E}$ STIMATES OF AUTISM SPECTRUM DISORDER (ASD) prevalence have ranged from $0.7 \%$ to $>2.5 \%$, with increases over time generally attributed to improved identification, especially among those without co-occurring intellectual disability (ID). ${ }^{1}$ Previous research in children has suggested that multiple factors affect age of initial diagnosis, with delayed diagnoses associated with a lack of language delay, minority status, low SES status, and co-occurring diagnoses such as ADHD and anxiety. ${ }^{2,3}$ The number of undiagnosed or misdiagnosed adults on the autism spectrum provides an additional area of concern. ${ }^{4-6}$ In adults, factors that complicate an initial diagnosis include difficulties obtaining a developmental history, high rates of co-occurring psychopathology, and learned camouflaging behaviors. ${ }^{7}$ Autistic individuals diagnosed in adulthood often have been either misdiagnosed with or have co-occurring psychiatric disorder diagnoses such as anxiety disorders, ADHD, mood disorders, and personality disorders. ${ }^{5,8,9}$ Misdiagnoses often result in missing services or supports. In many cases, it is essential to identify adults as being on the spectrum to access needed services. However, there is no standard diagnostic process for identifying ASD. ASD diagnoses are generally made after behavioral observation and interview on autistic features. The United Kingdom's National Institute for Health and Care Excellence recommends the use of multiple professionals and a comprehensive assessment of core ASD and related characteristics (e.g., daily functioning, commonly overlapping diagnoses such as anxiety, and other neurodevelopmental diagnoses) that can include standardized measures alongside interviewing. ${ }^{10}$ Best practices in ASD assessment suggest using multiple measures (interviews and questionnaires) and multiple reporters (e.g., self and parent/caregiver). ${ }^{11,12}$

An additional barrier to the diagnosis of adults on the spectrum is the comparative paucity of assessment tools that specifically diagnose ASD among adults. Relatively few measures have been modified, psychometrically tested, or developed specifically for adults. ${ }^{13,14}$ For example, previous research has questioned the "gold-standard" tools for diagnosing ASD, the Autism Diagnostic Observation Schedule (ADOS) ${ }^{15,16}$ and Autism Diagnostic Interview-Revised
$(\mathrm{ADI}-\mathrm{R})^{17}$ for their utility among adults on the spectrum. The ADOS-2 is a semistructured diagnostic assessment and is one of the most commonly used instruments for research and clinical use. It consists of five modules that are suited for different language and developmental levels, with Module 4 designed for adolescents and adults with fluent verbal speech. The ADI-R is a standardized clinical caregiver interview of autism characteristics during early development. Sensitivity (proportion of true positives identified; correctly diagnosed) and specificity (proportion of true negatives identified; correctly not diagnosed) measure diagnostic accuracy. The ADOS-2's sensitivity and specificity were classified at least $80 \%$ and $70 \%$, respectively, ${ }^{16}$ and a new algorithm consistent with Diagnostic and Statistical Manual of Mental Disorders, Fifth Edition (DSM-5) classification of ASD was found to be similarly effective. ${ }^{18}$ However, other studies have not had consistent findings (Table 1). A recent study of adults without ID found that the ADOS, but not ADI-R, was concordant with ASD diagnosis. ${ }^{19}$ Bastiaansen et al. ${ }^{14}$ found that the ADOS was not able to distinguish between adults on the spectrum and with schizophrenia without modification of the scoring algorithm, but it was able to reliably distinguish between ASD and both typical development and psychopathy. Another sample found that $50 \%$ of individuals with schizophrenia exceeded the cutoff for ASD. ${ }^{20}$ Among individuals at a community mental health center setting, $30 \%$ of individuals with psychosis were found to have scores above the cutoffs for ASD; however, $100 \%$ of previously diagnosed autistic individuals were correctly identified. ${ }^{21}$ In a sample of individuals without ID, the ADOS had the highest sensitivity and specificity among females and among individuals $>16$ years old. ${ }^{11}$ Adults with ID have also been shown to be overidentified with co-occurring ASD when using the ADOS. ${ }^{22}$ Overall, it has been suggested that behavioral and features that overlap with other disorders account for difficulties in relying on the ADOS as the sole diagnostic tool, and the possibility of "false positives" among other diagnostic populations remains an issue. ${ }^{14,22}$

There are also several self-report questionnaires currently used to diagnose ASD among adults. Baron-Cohen et al. ${ }^{23}$ developed the Autism Spectrum Quotient (AQ) primarily as a measure of broader autism phenotype characteristics, and for use only in conjunction with other measures as an ASD 
Table 1. Summary of Autism Diagnostic Observation Schedule, Autism Quotient, and Ritvo Autism AsPerger's Diagnostic Scale-Revised Findings

\begin{tabular}{|c|c|c|c|}
\hline Study & Population characteristics & Sensitivity (\%) & Specificity (\%) \\
\hline \multicolumn{4}{|l|}{ ADOS } \\
\hline Lord et al. ${ }^{15}$ & $\begin{array}{l}\text { Majority with ASD; small non-ASD sample with ID } \\
\text { and other psychiatric diagnoses }\end{array}$ & 90.0 & 93.0 \\
\hline Bastiaansen et al. ${ }^{14}$ & No ID; range of psychiatric diagnoses & 61.0 & 82.0 \\
\hline Hus and Lord ${ }^{18}$ & $\begin{array}{l}\text { Majority with ASD; adolescents and adults with ID } \\
\text { and other psychiatric diagnoses }\end{array}$ & 90.5 & 82.2 \\
\hline Pugliese et al. ${ }^{11}$ & Adolescents and adults without ID & 81.0 & 77.9 \\
\hline de Bildt et al. ${ }^{20}$ & $\begin{array}{l}\text { Adult males with ASD, schizophrenia, psychopathy, } \\
\text { and controls }\end{array}$ & 61.0 & 95.0 \\
\hline Maddox et al. ${ }^{21}$ & Adults in CMHC & 100.0 & 74.0 \\
\hline Fusar-Poli et al. ${ }^{19}$ & Adults referred to ASD clinic; no ID & 85.9 & 82.9 \\
\hline \multicolumn{4}{|l|}{ AQ } \\
\hline Baron-Cohen et al. $^{23}$ & $\begin{array}{l}\text { ASD without ID; controls from an undergraduate } \\
\text { college sample }\end{array}$ & 79.3 & 98.0 \\
\hline Ketelaars et al. ${ }^{26}$ & Adult outpatients with and without ASD; without ID & Not reported & Not reported \\
\hline Sizoo et al. ${ }^{12}$ & Adults with ASD and controls (AQ-28) & 57.0 & 70.0 \\
\hline Ashwood et al. ${ }^{25}$ & Adults referred to an ASD clinic for diagnosis & 77.0 & 29.0 \\
\hline \multicolumn{4}{|l|}{ RAADS-R } \\
\hline Ritvo et al. ${ }^{27}$ & $\begin{array}{l}\text { Adults with ASD, other psychiatric diagnoses, or } \\
\text { controls with no diagnosis }\end{array}$ & 97.0 & 100 \\
\hline Andersen et al. ${ }^{28}$ & Adults with ASD and controls & 90.7 & 92.9 \\
\hline Sizoo et al. ${ }^{1}$ & Adults with ASD and controls & 73.0 & 58.0 \\
\hline
\end{tabular}

ADOS, Autism Diagnostic Observation Schedule; AQ, Autism Spectrum Quotient; ASD, autism spectrum disorder; CMHC, Community Mental Health Center; ID, intellectual disability; RAADS-R, Ritvo Autism Asperger's Diagnostic Scale-Revised.

screening tool, although it has been utilized in research and clinical samples of autistic individuals. ${ }^{24}$ Comparisons of autistic individuals and a clinical outpatient sample found no significant differences between groups, ${ }^{25}$ and one study observed differences only on the communication subscale. ${ }^{26}$ Short forms of the AQ (28- and 10-item versions) found comparable levels of sensitivity (0.77-0.79) but low levels of specificity (0.36-0.41) among an ASD diagnosis-seeking Dutch sample. ${ }^{12}$

The Ritvo Autism Asperger's Diagnostic Scale-Revised $(\text { RAADS-R })^{27}$ was also developed as a self-report screening tool for ASD characteristics in individuals without cooccurring ID. Utilizing >700 autistic individuals at nine sites, Ritvo et al. found the RAADS-R sensitivity, specificity, and test-retest reliability to be in acceptable ranges. ${ }^{27}$ Furthermore, the RAADS-R was also found to have concurrent validity with the Social Responsiveness Scale, a self-report measure of ASD features. A Swedish translation of the RAADS-R also found good sensitivity, specificity, test-retest reliability, and correlation with participants who also completed the AQ. ${ }^{28}$ Similar sensitivity and specificity were observed in a Dutch sample of 210 adults in a diagnostic clinic. ${ }^{12}$

Previous research has suggested that the ability for current self-report measures to predict a diagnosis of ASD is lacking in an outpatient clinic setting. ${ }^{12}$ Self-report measures, in particular, may pose a difficulty for autistic individuals, because in many cases it can require comparison with other's internal states. ${ }^{29}$ One potential explanation for such disparities in self-report is alexithymia, difficulties in identifying and describing one's own emotions. Research has shown higher levels of alexithymia among autistic adults, ${ }^{30}$ and it has been shown to be uniquely associated with emotional impairment in ASD. ${ }^{31}$ An additional limitation of current self-report measures may be that non-ASD individuals designed them. Research concerning perspective taking among autistic adults has shown that they are able to recognize the effects of their ASD and that their family members are likely to rate ASD-related skills lower than their own self-ratings. ${ }^{32}$ A study comparing adults' AQ with parental report of developmental history found that only $44 \%$ of adults on the spectrum scored above the cutoffs. ${ }^{33}$ However, one study that assessed the predictive validity of two short forms of the AQ and the RAADS-R found high levels $(80 \%)$ of confirmation of ASD diagnosis among adults. ${ }^{12}$

The purpose of this study is to explore the correspondence between several adult ASD measures (AQ, RAADS-R, and ADOS) in diagnosing adults on the spectrum at an outpatient adult ASD diagnostic clinic. Although relying on one measure to make an ASD diagnosis is not a recommended approach, it is important to investigate the association between individual measures and a clinical diagnosis. Previous studies have compared self-report measures or assessed the validity of the ADOS-2 or other measures among groups of adults with different diagnoses, but this article is one of the first to look at both the ADOS-2 and self-report measures in a large group of adults seeking an ASD diagnosis. Based upon previous research, we expected that the ADOS would have greater correspondence to final diagnosis than either AQ or RAADS-R.

\section{Methods}

\section{Participants}

Ninety-three adults assessed through an outpatient adult ASD diagnostic clinic at a university medical center setting were participants in this study. The participants consisted of 
all evaluations at the clinic from 2010 to 2013. Participants were either self-referrals or were referred by another medical provider and ranged in age from 18 to 61 years old. The majority of the participants were male $(n=67 ; 72.04 \%)$ and Caucasian/white (51.61\%). Ages ranged from 18 to 61 years $(\mathrm{M}=35.34)$. Many had previous diagnoses, including ADHD, specific learning disabilities (e.g., impairment in reading, writing, or math), and anxiety disorders (Table 2). Of the 93 individuals, 3 held a previous diagnosis of ID (all of whom were found to not have an ASD diagnosis).

\section{Procedure}

We conducted a retrospective review of medical records from the diagnostic clinic. The first author deidentified cli- ents' data and entered it into a database. The University of Pittsburgh Institutional Review Board approved the project.

As part of usual care, when prospective clients called the clinic to schedule an appointment, the second author mailed them a packet containing the demographics questionnaire, AQ, and RAADS-R. The clients either completed these measures before coming in for their first appointment, with a clinician reviewing the items with the clients at the first appointment, or they completed them at the first appointment with the assistance of the clinician (e.g., reading items aloud, defining vocabulary, or explaining vague items) if needed. At the first appointment, clients completed an in-depth clinical interview with particular focus on their developmental history. In addition, if the client consented to do so, parents, spouses, and other family members were welcomed to

Table 2. Demographics and Sample Descriptives

\begin{tabular}{|c|c|c|c|c|}
\hline & $A S D \operatorname{group}(\mathrm{n}=31)$ & Non-ASD group $(\mathrm{n}=62)$ & Total sample $(\mathrm{n}=93)$ & $\mathrm{p}$ \\
\hline Male & $22(71 \%)$ & $45(72.6 \%)$ & $67(72 \%)$ & 0.87 \\
\hline Age & & & & 0.52 \\
\hline Under 20 & 2 & 5 & 7 & \\
\hline $20-29$ & 13 & 21 & 34 & \\
\hline $30-39$ & 5 & 13 & 18 & \\
\hline $40-49$ & 8 & 10 & 18 & \\
\hline $50+$ & 3 & 13 & 17 & \\
\hline Race & & & & 0.48 \\
\hline Asian & 0 & $1(1.6 \%)$ & $1(1.1 \%)$ & \\
\hline Black & $1(3.2 \%)$ & $2(3.2 \%)$ & $3(3.2 \%)$ & \\
\hline Hispanic/Latino & 0 & 0 & 0 & \\
\hline White & $14(45.2 \%)$ & $34(54.8 \%)$ & $48(51.6 \%)$ & \\
\hline Not reported & $14(45.2 \%)$ & $25(40.3 \%)$ & $39(41.9 \%)$ & \\
\hline Level of education & & & & -0.16 \\
\hline$<$ High school & 0 & $4(6.4 \%)$ & 4 & \\
\hline High school & $13(41.9 \%)$ & $22(35.5 \%)$ & 35 & \\
\hline Some postsecondary & $11(35.4 \%)$ & $14(22.5 \%)$ & 25 & \\
\hline College & $5(16.1 \%)$ & $11(17.7 \%)$ & 16 & \\
\hline Graduate & $2(6.4 \%)$ & $8(12.8 \%)$ & 10 & \\
\hline Unknown & 0 & $3(4.8 \%)$ & 3 & \\
\hline Special education received & $6(19.4 \%)$ & $8(12.9 \%)$ & $14(15.1 \%)$ & 0.82 \\
\hline Employed & $10(32.3 \%)$ & $20(32.3 \%)$ & $30(31.9 \%)$ & 1.0 \\
\hline Married & $4(12.9 \%)$ & $14(22.6 \%)$ & $18(19.4 \%)$ & 0.26 \\
\hline Has child(ren) & $6(19.4 \%)$ & $20(32.3 \%)$ & $26(28 \%)$ & 0.16 \\
\hline \multicolumn{5}{|l|}{ Previous diagnosis } \\
\hline ADHD & 1 & 10 & 11 & \\
\hline SLD & 7 & 2 & 9 & \\
\hline ID & 0 & 3 & 3 & \\
\hline Anxiety/OCD & 17 & 11 & 28 & \\
\hline Depression & 13 & 13 & 26 & \\
\hline Bipolar & 8 & 0 & 8 & \\
\hline Psychotic disorder & 1 & 1 & 2 & \\
\hline Substance abuse & 3 & 0 & 3 & \\
\hline Personality disorder & 3 & 0 & 3 & \\
\hline \multirow[t]{2}{*}{ Other } & 1 & 5 & 6 & \\
\hline & $M(S D)$ & $M(S D)$ & Hedge's g & \\
\hline RAADS-R total & $109.00(51.02)$ & 94.69 (49.59) & 0.29 & \\
\hline AQ total & $30.65(5.89)$ & $33.10(5.33)$ & & \\
\hline ADOS total & $8.68(3.21)$ & $6.69(4.35)$ & 0.49 & \\
\hline$\%$ Autism cutoff & $12(38.7 \%)$ & $9(14.5 \%)$ & & \\
\hline$\%$ Spectrum cutoff & $11(35.5 \%)$ & $12(29.4 \%)$ & & \\
\hline
\end{tabular}

ADHD, attention-deficit/hyperactivity disorder; OCD, obsessive-compulsive disorder; SLD, specific learning disorder (in reading, writing, and/or math). 
participate in the clinical interview, and, in some cases, were interviewed by phone on another date. When other individuals were interviewed, questions focused on developmental history and current autistic characteristics.

The ADOS was administered at a second meeting with a second trained master's- or doctoral-level clinician. All clinicians attended ADOS clinical training before administering the ADOS at the clinic. ADOS clinicians also had access to consult with research-trained ADOS clinicians as needed.

Afterward, the two clinicians met together to conceptualize and determine diagnosis. Clinicians rereviewed all available information (interviews, measures, and records if available) and made a best estimate clinical ASD diagnosis based on Diagnostic and Statistical Manual of Mental Disorders, 4th Edition, Text Revision (DSM-IV-TR) criteria. Although clinicians referenced the ADOS, AQ, and RAADS-R scores in making a clinical diagnosis, they were not solely used to assign a diagnosis. Instead, clinicians evaluated scores and report of past and current autistic features to DSM-IV-TR criteria to make an ASD diagnosis. Previous diagnoses (e.g., when a client held a prior diagnosis of ADHD) were typically upheld unless ASD was an exclusion. When clinicians noted characteristics of another diagnosis during the evaluation that fit diagnostic criteria, such as social phobia or ADHD, and ASD was not diagnosed, these diagnoses were made.

\section{Measures}

\section{Demographic questionnaire}

Clinicians in this clinic developed a questionnaire regarding demographics and developmental, medical, and familial history, which we used for this study. In cases wherein the client did not complete the demographic interview, clinicians obtained information during the clinical interview with the client whenever possible.

\section{Autism Spectrum Quotient}

The AQ is a 50-item self-report screening measure of ASD characteristics. Although the AQ's authors originally developed to identify ASD characteristics in neurotypical samples, past research has also used the AQ as a screening instrument for ASD. ${ }^{23,34}$ The measure consists of 50 items assessing areas of social skills, attention switching, attention to detail, communication, and imagination. When filling out the measure, people rank the items on a 4-point Likert scale ("definitely agree to definitely disagree"), which are then scored 0 or 1. Higher scores indicate more ASD characteristics. We used the clinical cutoff' of $\geq 32$ that Baron-Cohen et al. ${ }^{23}$ identified. They showed that the AQ subscales have internal consistency between 0.60 and 0.80 , and test-retest reliability of $0.70 .{ }^{23}$ In this sample, internal consistency (reliability) was $\alpha=0.82$. A typical rule of thumb for reliability is $>0.8$ is good, $>0.7$ acceptable, $>0.6$ questionable, and $<0.6$ poor. $^{35}$

\section{Autism Diagnostic Observation Schedule, Module 4 (ADOS)}

The ADOS is a semistructured observational assessment used to diagnose ASD, where higher scores indicate more ASD features. ${ }^{15,16}$ The ADOS is an assessment that com- prises several structured activities and interview questions that are designed to elicit behaviors associated with ASD. Codes fall into communication, reciprocal social interaction, imagination/creativity, and stereotyped behaviors and restricted interests domains, in line with DSM-IV-TR criteria for ASD. The ADOS has two cutoffs; 10 and above for autism and 7 and above for autism spectrum. In this study, we used seven and above as the ASD cutoff for clinical diagnoses. For this study, clinicians used both the ADOS and ADOS-2. The ADOS-2 Module 4 did not change in administration and scoring from the first edition. In laboratories and university clinics, sensitivity is at least $80 \%$ and specificity is at least $70 \% .^{11,18} \mathrm{We}$ did not use the newer version of the algorithm $^{18}$ in this study to maintain consistent scores across participants independent of DSM criteria used for diagnosis.

\section{Ritvo Autism Asperger's Diagnostic Scale-Revised}

Ritvo et al. designed the RAADS-R as a self-report measure to screen for the presence of ASD characteristics in adults based on DSM-IV-TR criteria. ${ }^{27}$ It consists of 80 items in four domains (social interaction, language, circumscribed interests, and sensory motor features). Individuals using the measure rated themselves on a 4-point Likert scale ("never true" to "true now and when I was young"), with 17 items reverse coded. Items on this scale are also life span focused. The cutoff used in this study is $\geq 65$. $^{27}$ Ritvo et al. ${ }^{27}$ found concurrent validity with the Social Responsiveness ScaleAdult $(95.59 \%)$. Internal consistency ranged from 0.87 to 0.95 on the subscales, and test-retest reliability of $0.99 .^{27}$ Reliability for this sample is $\alpha=0.95$.

\section{Analysis}

The authors analyzed the data using IBM SPSS Statistics Version 24.0, running correlations, corrected by z-scores, and independent sample $t$-tests to compare the groups (diagnosed ASD and not diagnosed) on gender, age, ethnicity, current employment status, and ASD measures. Authors calculated measure-specific sensitivity and specificity, and receiver operating characteristic curves, which are plots of the true positive rate (sensitivity) against false positive rate (onespecificity) across the range of all possible cutoffs. The area under the curve (AUC) is a measure of test accuracy, where higher scores (approaching 1.00) signify higher accuracy of the measure. AUC can also be used to determine the optimal cutoff for a measure. One common method for determining optimal cutoffs is to find the cutoff with highest sum of sensitivity and specificity, ${ }^{36}$ which the authors used in this study.

\section{Results}

Of the 93 individuals in this study, clinicians diagnosed $31(33.33 \%)$ of them with ASD. Age, gender, ethnicity, and employment status did not significantly differ between groups, and correlations between those diagnosed with ASD and those not diagnosed on these demographic variables were not significantly different, as observed by Fisher's Z-transformation (all Z<1.05). Both the ADOS $\left(t_{(83)}=-2.23\right.$, $p=0.03 ; g=0.49)$ and AQ $\left(t_{(91)}=-2.02, p=0.046 ; g=0.44\right)$ differed significantly between those diagnosed with ASD and those who were not diagnosed, but the RAADS$\mathrm{R}$ total score $\left(t{ }_{(91)}=-1.30, p=0.20 ; g=0.29\right)$ did not 
Table 3. Study Results

\begin{tabular}{lccccc}
\hline & AUC & 95 CI & Typical cutoffloptimal cutoff & Sensitivity & Specificity \\
\hline ADOS & 0.694 & $0.577-0.810$ & 7 & 0.68 & 0.70 \\
& & & 8.5 & 0.645 & 0.759 \\
AQ & 0.400 & $0.280-0.519$ & 32 & 0.55 & 0.34 \\
\multirow{2}{*}{ RAADS-R } & & & 33.5 & 0.45 & 0.52 \\
& 0.581 & $0.455-0.707$ & 90 & 0.65 & 0.44 \\
& & & 129 & 0.61 & 0.53 \\
& & & & & 0.52 \\
\hline
\end{tabular}

AUC, area under the curve; CI, confidence interval.

significantly differ between groups. However, the AQ was significantly higher for the non-ASD group than for the ASD group.

The AUC was the following for each measure: $\mathrm{ADOS}=0.69$ (95\% confidence interval $[\mathrm{CI}] 0.58-0.81$ ), RAADS-R $=0.58$ (95\% CI 0.46-0.72), and AQ $=0.40$ (95\% CI 0.28-0.52) (Table 3 and Figs. 1-3). An ADOS total communicationsocial score of 7.50 was associated with a sensitivity of 0.68 and a specificity of 0.70 , which is slightly higher than the clinical cutoff (7) of the ADOS. The ideal cutoff for this sample is 8.50 , associated with sensitivity of 0.65 and specificity of 0.76 . On the RAADS-R, a score of 64.50 (clinical cutoff $=65$ ) was associated with sensitivity of 0.65 and specificity of 0.44 , whereas a RAADS-R score of 90 was associated with sensitivity of 0.61 and specificity of 0.53 . The cutoff for this sample, 129 , was associated with sensitivity of 0.52 and specificity of 0.73 . For the $A Q$, where a clinical cutoff is 32 is commonly used, the sensitivity associated for a score of 31.50 was 0.55 and specificity was 0.34 . The optimal clinical cutoff for this sample was 33.50 , associated with 0.45 sensitivity and 0.52 specificity.

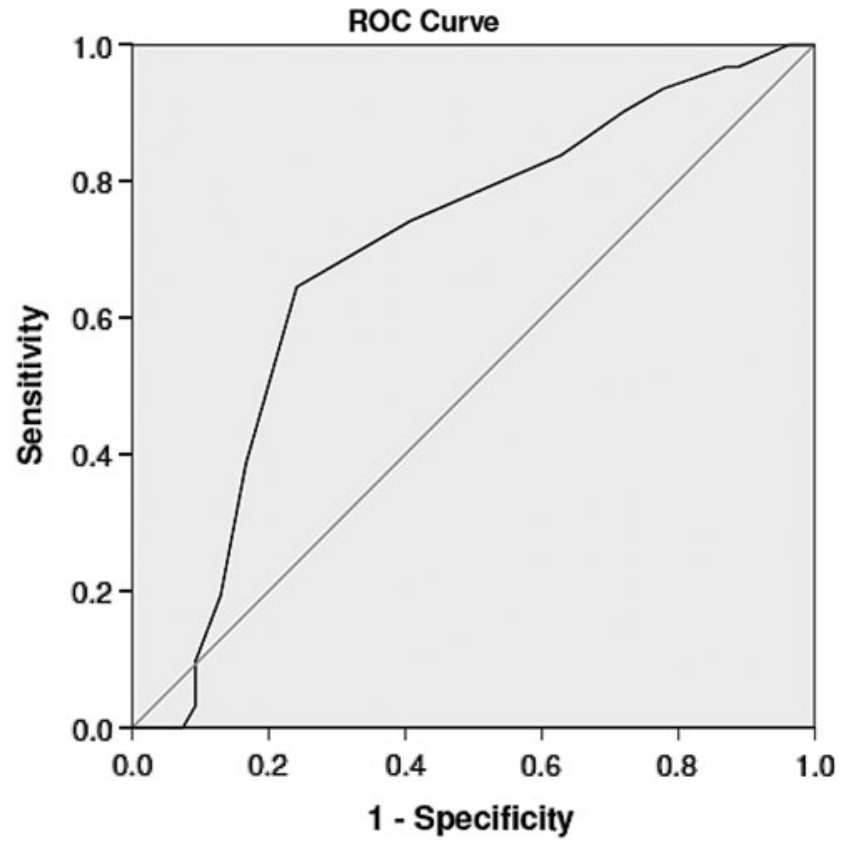

FIG. 1. ADOS ROC curve. ADOS, Autism Diagnostic Observation Schedule; ROC, receiver operating characteristic.

\section{Discussion}

The aim of this study was to examine the predictive validity of ASD measures against clinical diagnosis in an outpatient adult ASD clinic. Although ADOS scores were significantly higher among the participants diagnosed with ASD, measures of sensitivity, specificity, and AUC were fair at best. Consistent with previous research, ${ }^{1,20-22}$ the accuracy of the ADOS was lower than the reported sensitivity of $80 \%$ and specificity of $70 \%$ found by the developers. ${ }^{15}$ Furthermore, this study is consistent with previous research at an ASD clinic, ${ }^{12}$ as the AQ and RAADS-R were both found to not be predictive of a diagnosis. AQ scores were even higher in the group without ASD than in the group with ASD. These differences could be due, in part, to increased heterogeneity of subsequent samples compared with the original ADOS studies. Many measure development studies consist of individuals with a previous ASD diagnosis and smaller control samples without any psychiatric diagnoses, which can cause spectrum bias, leading to higher specificity and sensitivity than outpatient and community samples. It is also possible that these self-report questionnaires may be more useful for

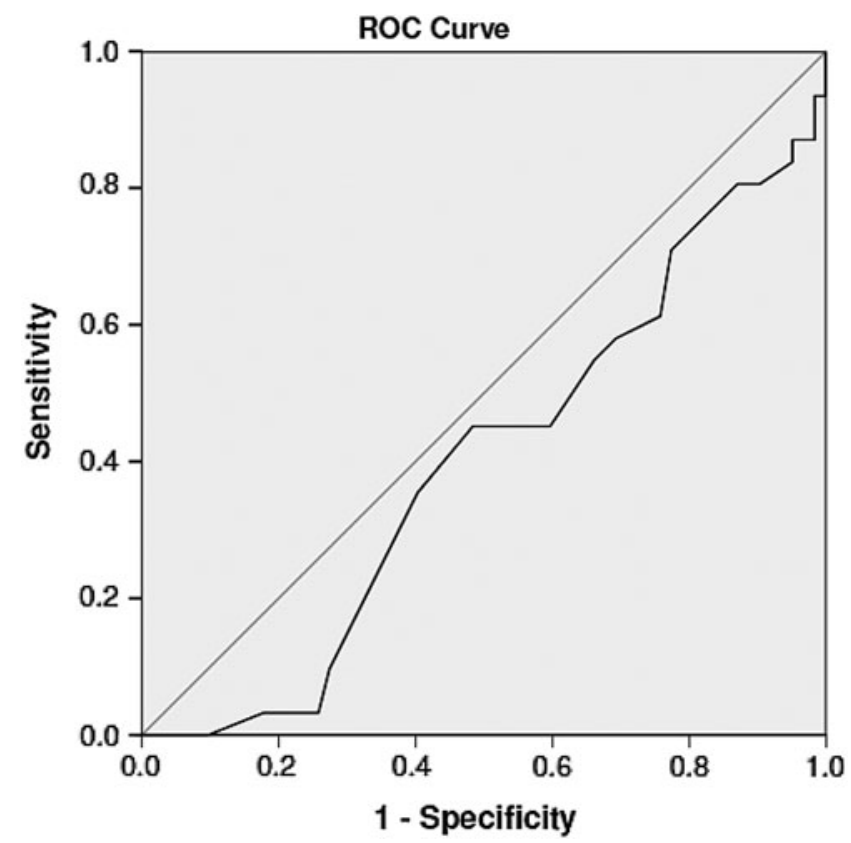

FIG. 2. AQ ROC curve. AQ, Autism Spectrum Quotient. 


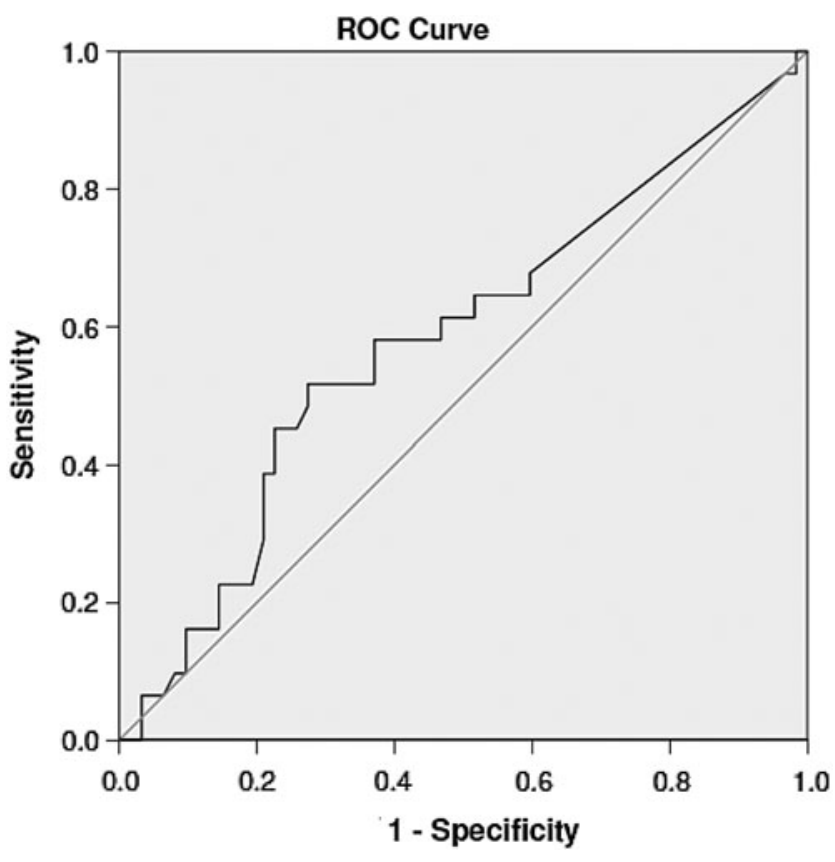

FIG. 3. RAADS-R ROC curve. RAADS-R, Ritvo Autism Asperger's Diagnostic Scale-Revised.

studying autism features across general population samples rather than for diagnostic purposes. Adults who receive an initial ASD diagnosis as adults (often through these outpatient clinics) are also likely to have milder or more complex presentations and may have learned camouflaging or other strategies that can challenge identification of core aspects of ASD. ${ }^{7}$

There are limitations to consider in this study. Two clinicians made each participant's diagnosis after review of their records, as well as their AQ, RAADS-R, and ADOS by both the clinicians, so the clinicians were not blind to history or measures. A separate evaluator who is naive to final diagnosis, as well as the measures used, could have provided a more objective measure of diagnostic status, the lack of which is a sizable limitation to this study. Future studies should consider a naive evaluator to improve the generalizability of findings across clinics. Relatedly, the authors of this study were unable to examine detection of missed diagnosis ("false negatives"). It is possible that the ADOS is most highly associated with diagnostic decisions due to the amount of time that a clinician spent administering the ADOS, the expectation that the ADOS is the "gold standard" measure, and that more information is collected during the ADOS for a potential ASD diagnosis than in a questionnaire.

The relatively low rate of ASD diagnoses (33\%) also provides another potential explanation for the results of this study. This rate is half of Sizoo et al.'s ${ }^{12}$ study, where $\sim 66 \%$ of patients referred for an ASD evaluation were found to have ASD. An individual did not need a referral from another medical professional to obtain an evaluation at the clinic, which may also affect the population served there as compared with other clinics. Clinicians did not assess for other diagnoses, including ADHD, mood and anxiety disorders, and personality disorders, as well as intelligence quotient (IQ), and thus the diagnostic makeup of the participants who were not diagnosed with ASD is limited to their self-report of prior diagnoses and IQ results (if there was previous testing). Previous research has shown that other diagnoses, as well as other demographic factors such as college major or occupation, can result in higher AQ scores. ${ }^{23} \mathrm{~A}$ better understanding of the nondiagnosed sample could lead to identifying which overlapping characteristics or traits, if any, may lead a person without ASD to consider the possibility of an ASD diagnosis.

Future studies should further explore the characteristics of screening and diagnostic tools for adults. It is quite understandable that measures for autistic individuals, much like any other diagnostic tool, have been developed with carefully curated samples with pre-existing diagnoses. These samples provide evidence of sensitivity and specificity. However, these samples are not representative of all those suspected of ASD. In particular, it is vital to examine these measures in real-world settings, such as in outpatient clinics. Clinicians in these settings may also vary more in terms of ADOS training; for example, research-trained ADOS clinicians attend a longer and more advanced ADOS training and have their ADOS administration and scoring reviewed for reliability, whereas clinicians who administer the ADOS for clinical use in outpatient clinics may not have this level of intensive training. ${ }^{15}$ Future research should also assess other measures, such as the Social Responsiveness Scale (SRS-2), ${ }^{37}$ and parent/other-report measures in clinic settings to better understand their diagnostic validity. Furthermore, this study highlights the importance of developing new and tailoring existing measures for adults across cognitive functioning, gender, age, and co-occurring psychiatric conditions. ${ }^{11} \mathrm{Al}-$ though measures to date have typically been developed using samples of individuals with and without ID, this study may indicate that such measures perform less accurately in a sample wherein a majority do not have co-occurring ID. Future measure development should also involve autistic individuals and their viewpoints on how best to assess and describe ASD characteristics. ${ }^{38}$ The Interagency Autism Coordinating Committee at the U.S. National Institutes of Health has also pointed to the development of adult-specific diagnostic and treatment outcome measures. ${ }^{6}$

Results from this study have several implications for diagnosing adults on the spectrum. As other studies have previously suggested, clinicians should exercise caution when using these existing diagnostic tools with adults on the spectrum. Utilizing more than one method of assessment, including parent-/other- report of history, ADOS, clinical interviews, and questionnaires, is necessary to give a fuller more accurate picture. In accordance with previous studies, this study does not recommend sole reliance upon the ADOS or existing selfreport measures. In particular, the AQ was initially designed to capture broader autism traits in nonclinical samples, ${ }^{23}$ and the extant literature is mixed concerning the ability to use it in a diagnostic context. Given the need for services that are adapted for adults on the spectrum to address problematic outcomes, the ability to reliably diagnose adults will assist individuals in gaining access to these services and supports.

\section{Acknowledgments}

This project was funded through a grant from the Bureau of Autism Services, Department of Human Services, Commonwealth of Pennsylvania to the Western Regional Autism Services, Education, Resources, Training (ASERT) 
Collaborative at Western Psychiatric Institute and Clinic at the University of Pittsburgh Medical Center. The Pennsylvania Bureau of Autism Services was not involved in the study design, analysis, or decision to submit this article.

\section{Authorship Confirmation Statement}

All authors jointly conceived of the study. C.M.C. supervised entry of medical records and created the data set, ran analyses, and drafted the article. R.D.C. and J.J.M. edited and approved the article. All authors have approved this article, and this article is submitted solely to Autism in Adulthood and is not published, in press, or submitted elsewhere.

\section{Author Disclosure Statement}

No competing financial interests exist.

\section{References}

1. Lyall K, Croen L, Daniels J, et al. The changing epidemiology of autism spectrum disorders. Annu Rev Public Health. 2017;38(1):81-102.

2. Mandell DS, Novak MM, Zubritsky CD. Factors associated with age of diagnosis among children with autism spectrum disorders. Pediatrics. 2005;116(6):1480-1486.

3. Soke GN, Maenner MJ, Christensen D, Kurzius-Spencer M, Schieve LA. Prevalence of co-occurring medical and behavioral conditions/symptoms among 4- and 8-year-old children with autism spectrum disorder in selected areas of the United States in 2010. J Autism Dev Disord. 2018; 48(8):2663-2676.

4. Nylander L, Gillberg C. Screening for autism spectrum disorders in adult psychiatric out-patients: A preliminary report. Acta Psychiatr Scand. 2001;103(6):428-434.

5. Bresnahan M, Li G, Susser E. Hidden in plain sight. Int $J$ Epidemiol. 2009;38(5):1172-1174.

6. Interagency Autism Coordinating Committee. Strategic Plan for Autism Spectrum Disorder. Bethesda, MD; 2016. https://iacc.hhs.gov/publications/strategic-plan/2012 Accessed November 8, 2018.

7. Lai MC, Baron-Cohen S. Identifying the lost generation of adults with autism spectrum conditions. Lancet Psychiatry. 2015;2(11):1013-1027.

8. Luciano CC, Keller R, Politi P, et al. Misdiagnosis of high functioning autism spectrum disorders in adults: An Italian case series. Autism Open Access. 2014;04(02). DOI: 10.4172/2165-7890.1000131.

9. Geurts HM, Jansen MD. A retrospective chart study: The pathway to a diagnosis for adults referred for ASD assessment. Autism. 2012;16(3):299-305.

10. Pilling S, Baron-Cohen S, Megnin-Viggars O, Lee R, Taylor C. Recognition, referral, diagnosis, and management of adults with autism: Summary of NICE guidance. $B M J$. 2012;344:e4082.

11. Pugliese CE, Kenworthy L, Bal VH, et al. Replication and comparison of the newly proposed ADOS-2, Module 4 algorithm in ASD without ID: A multi-site study. J Autism Dev Disord. 2015;45(12):3919-3931.

12. Sizoo BB, Horwitz EE, Teunisse JJ, et al. Predictive validity of self-report questionnaires in the assessment of autism spectrum disorders in adults. Autism. 2015;19(7):842-849.

13. Matson JL, Neal D. Diagnosing high incidence autism spectrum disorders in adults. Res Autism Spectr Disord. 2009;3(3):581-589.
14. Bastiaansen JA, Meffert H, Hein S, et al. Diagnosing autism spectrum disorders in adults: The use of Autism Diagnostic Observation Schedule (ADOS) Module 4. J Autism Dev Disord. 2011;41(9):1256-1266.

15. Lord C, Risi S, Lambrecht L, et al. The autism diagnostic observation schedule-generic: A standard measure of social and communication deficits associated with the spectrum of autism. J Autism Dev Disord. 2000;30(3):205-223.

16. Lord C, Rutter M, DiLavore PC, Risi S, Gotham K, Bishop SL. Autism Diagnostic Observation Schedule-2. Los Angeles: Western Psychological Services; 2012.

17. Lord C, Rutter M, Le Couteur A. Autism Diagnostic Interview-Revised: A revised version of a diagnostic interview for caregivers of individuals with possible pervasive developmental disorders. J Autism Dev Disord. 1994; 24(5):659-685.

18. Hus V, Lord C. The Autism Diagnostic Observation Schedule, Module 4: Revised algorithm and standardized severity scores. J Autism Dev Disord. 2014;44(8):19962012.

19. Fusar-Poli L, Brondino N, Rocchetti M, et al. Diagnosing ASD in adults without ID: Accuracy of the ADOS-2 and the ADI-R. J Autism Dev Disord. 2017;47(11):3370-3379.

20. de Bildt A, Sytema S, Meffert H, Bastiaansen JA. The Autism Diagnostic Observation Schedule, Module 4: Application of the revised algorithms in an independent, welldefined, Dutch Sample ( $n=93)$. J Autism Dev Disord. 2016; 46(1):21-30.

21. Maddox BB, Brodkin ES, Calkins ME, et al. The accuracy of the ADOS-2 in identifying autism among adults with complex psychiatric conditions. J Autism Dev Disord. 2017;47(9):2703-2709.

22. Sappok T, Diefenbacher A, Budczies J, et al. Diagnosing autism in a clinical sample of adults with intellectual disabilities: How useful are the ADOS and the ADI-R? Res Dev Disabil. 2013;34(5):1642-1655.

23. Baron-Cohen S, Wheelwright S, Skinner R, Martin J, Clubley E. The autism-spectrum quotient (AQ): Evidence from Asperger syndrome/high-functioning autism, males and females, scientists and mathematicians. J Autism Dev Disord. 2001;31(1):5-17.

24. Ruzich E, Allison C, Smith P, et al. Measuring autistic traits in the general population: A systematic review of the autism-spectrum quotient (AQ) in a nonclinical population sample of 6,900 typical adult males and females. Mol Autism. 2015;6(1):2.

25. Ashwood KL, Gillan N, Horder J, et al. Predicting the diagnosis of autism in adults using the autism-spectrum quotient (AQ) questionnaire. Psychol Med. 2016;46(12): 2595-2604.

26. Ketelaars C, Horwitz E, Sytema S, et al. Brief report: Adults with mild autism spectrum disorders (ASD): Scores on the autism spectrum quotient (AQ) and comorbid psychopathology. J Autism Dev Disord. 2008;38(1):176-180.

27. Ritvo RA, Ritvo ER, Guthrie D, et al. The Ritvo Autism Asperger Diagnostic Scale-Revised (RAADS-R): A scale to assist the diagnosis of Autism Spectrum Disorder in adults: An international validation study. J Autism Dev Disord. 2011;41(8):1076-1089.

28. Andersen LMJ, Näswall K, Manouilenko I, et al. The Swedish version of the Ritvo autism and asperger diagnostic scale: Revised (RAADS-R). A validation study of a rating scale for adults. J Autism Dev Disord. 2011;41(12): 1635-1645. 
29. Pearl AM, Edwards EM, Murray MJ. Comparison of selfand other-report of symptoms of autism and comorbid psychopathology in adults with autism spectrum disorder. Contemp Behav Health Care. 2016;2(1):1-8.

30. Berthoz S, Hill EL. The validity of using self-reports to assess emotion regulation abilities in adults with autism spectrum disorder. Eur Psychiatry. 2005;20:291-298.

31. Bird G, Cook R. Mixed emotions: The contribution of alexithymia to the emotional symptoms of autism. Transl Psychiatry. 2013;3(7):e285-e288.

32. Heasman B, Gillespie A. Perspective-taking is two-sided: Misunderstandings between people with Asperger's syndrome and their family members. Autism. 2017;22(6): 740-750.

33. Bishop SL, Seltzer MM. Self-reported autism symptoms in adults with autism spectrum disorders. J Autism Dev Disord. 2012;42(11):2354-2363.

34. Baron-Cohen S, Wheelwright S, Robinson J, WoodburySmith M. The Adult Asperger Assessment (AAA): A diagnostic method. J Autism Dev Disord. 2005;35(6):807-819.
35. George D, Mallery P. SPSS for Windows Step by Step: A Simple Guide and Reference. 11.0 Update. 4th ed. Boston, MA: Allyn \& Bacon; 2003.

36. Krzanowski WJ, Hand DJ. ROC Curves for Continuous Data. New York: Chapman \& Hall/CRC; 2009.

37. Constantino JN, Gruber CP. Social Responsiveness Scale2nd Edition (SRS-2). Torrance, CA: Western Psychological Services; 2012.

38. Ekblad L. Autism, personality, and human diversity: Defining neurodiversity in an iterative process using Aspie Quiz. SAGE Open. 2013;3:1-14.

Address correspondence to: Caitlin M. Conner, PhD ASERT Collaborative Western Region 1011 Bingham Street Pittsburgh, PA 15203

Email: connercm2@upmc.edu 